Review began 02/23/2022 Review ended 02/23/2022 Published 02/27/2022

๑) Copyright 2022

Gupta et al. This is an open access article distributed under the terms of the Creative Commons Attribution License CC-BY 4.0. which permits unrestricted use, distribution, and reproduction in any medium, provided the original author and source are credited.

\section{Use of Sotrovimab in a Pregnant Patient With COVID-19 Infection}

\author{
Isha Gupta 1, 2, 3 , Esther S. Arguello Perez ${ }^{4}$ \\ 1. Nephrology, Middletown Medical, Middletown, USA 2. Nephrology, Garnet Health Medical Center, Middletown, USA \\ 3. Internal Medicine/Nephrology, Touro College of Osteopathic Medicine, Middletown, USA 4. Infectious Disease, \\ BronxCare Health System, New York, USA
}

Corresponding author: Isha Gupta, guptaisha1984@gmail.com

\begin{abstract}
For individuals with mild-to-moderate coronavirus disease 2019 (COVID-19, caused by severe acute respiratory syndrome coronavirus 2 [SARS-CoV-2]), monoclonal antibodies (MOABs) are known to prevent progression of the disease and hospitalization. Pregnant women, who are at an increased risk of severe COVID-19 infection, have been significantly underrepresented in studies for MOAB treatments, especially sotrovimab. Specifically, there has only been one case reported of a pregnant woman using sotrovimab successfully. We report a second such patient - an unvaccinated 21-year-old, COVID-19-positive, 16-week pregnant woman who was followed closely over the next 60 days post-MOAB infusion. We noted prevention in the progression of the disease and hospitalization without any fetal/pregnancy-related complications.
\end{abstract}

Categories: Obstetrics/Gynecology, Infectious Disease, Therapeutics

Keywords: monoclonal antibodies, monoclonal antibody, sars-cov-2, omicron, pregnancy, coronavirus 2019, sotrovimab, covid-19, pregnant

\section{Introduction}

The Centers for Disease Control and Prevention (CDC) has reported 173,508 cases and 274 deaths of pregnant women due to coronavirus disease 2019 (COVID-19) in the United States between January 22nd 2020 to February 14th 2022 [1]. Pregnancy is a known risk factor for severe illness from COVID-19 infection [2]. The risk of ICU admission, requirement of invasive mechanical ventilation and death has been reported significantly higher amongst pregnant people compared to non-pregnant people [3]. There is also an increased risk of adverse obstetric outcomes amongst pregnant people with COVID-19, such as preterm or stillbirth $[4,5]$.

Within the general patient population, the activity of monoclonal antibodies (MOABs) against the spike protein of COVID-19 has been reported to decrease the risk of hospitalization and death [6-8]. On November 9th 2020, Emergency Use Authorization (EUA) was first issued to use MOABs in COVID-19 patients at risk of progression of the mild-to-moderate disease to a more severe form [9]. On May 14th 2021, the FDA included pregnancy as a qualifying condition for the use of these antibodies. With the rise of Omicron variant in December 2021, sotrovimab became the only MOAB effective against COVID-19 infection in nonhospitalized patients at the time [10]. Upon review of data in PubMed until February 20th 2022, only one case report about sotrovimab use in a COVID-19 pregnant patient has been described [11]. The case below outlines the second such case.

\section{Case Presentation}

The patient is an unvaccinated 21-year-old, 16-week pregnant woman, with a medical history of mild intermittent asthma. She first noticed symptoms on November 30th 2021, while she officially tested positive on December 6th (Table 1). During the follow-up telemedicine visit on December 7th, the patient revealed that while she was not experiencing fever, shortness of breath or a drop in oxygen saturation level below 95\% (self-monitored), she had been dealing with cough, nausea, sore throat, decreased sense of smell, chest congestion and headaches for the past week. 


\section{Cureus}

\begin{tabular}{|c|c|c|}
\hline TEST & RESULT & REFERENCE RANGE \\
\hline SARS-CoV-2 RT-PCR & Positive & Negative \\
\hline ORF1a gene & 20.2 (CT Values) & (CT Values) Not detected $<10$ or $>38$ Detected $10.0-38.0$ \\
\hline N gene & 20.6 (CT Values) & (CT Values) Not detected $<10$ or $>38$ Detected $10.0-38.0$ \\
\hline
\end{tabular}

\section{TABLE 1: COVID-19 testing}

SARS-CoV-2: severe acute respiratory syndrome coronavirus 2, RT-PCR: reverse transcription polymerase chain reaction, CT: cycle threshold

Given the risk factors of pregnancy, her vaccination status and the presence of mild symptoms of COVID-19, the patient was offered MOAB infusion. After consulting with her obstetrician, the patient accepted the infusion treatment. On December 8th 2021, the patient received sotrovimab infusion $(500 \mathrm{mg})$ mixed with normal saline over a 30-minute period. Her vitals were monitored through the infusion process and for another hour after it (Table 2). She tolerated it without any side effects.

\begin{tabular}{|c|c|c|c|c|c|}
\hline Stage of treatment & Temp (F) & $\mathrm{BP}(\mathrm{mmHg})$ & Pulse & RR & Oxygen sat. (\%) \\
\hline Pre-infusion & 98.7 & $144 / 68$ & 73 & 20 & 100 \\
\hline During-infusion & 98.9 & $134 / 69$ & 75 & 24 & 100 \\
\hline Post-infusion & 99.6 & $131 / 72$ & 94 & 25 & 99 \\
\hline
\end{tabular}

TABLE 2: Vital Signs monitored during the monoclonal antibody (MOAB) treatment

The patient had a telemedicine follow-up the next day, when she reported a significant improvement in her cough, nausea, headaches, and fatigue and felt only mild chest congestion at that time. She was also followed up on Day 14, Day 30, and Day 60 post infusion. The patient reported resolution of her symptoms and confirmed that she did not need any urgent care or emergency room visits or hospitalization during the follow-up period. No fetal or pregnancy-related complications were noted by her obstetrician, during her two monthly follow-ups since the infusion.

\section{Discussion}

On March 11th 2020, the World Health Organization (WHO) declared COVID-19 to be a global pandemic, its first such designation since declaring H1N1 influenza a pandemic in 2009. The pandemic has claimed about 5.8 million lives worldwide, out of which 921,984 deaths have been reported in the United States alone, as per the WHO database on February 18th, 2022. In the initial phase of the pandemic, different drugs and therapies like ivermectin, convalescent plasma, hydroxychloroquine/chloroquine, nitazoxanide etc. were used to treat COVID-19 infection, but their use was soon dissuaded by COVID-19 Treatment Guidelines Panel. Development of MOABs marked a major breakthrough in management of SARS-CoV-2, leading to Emergency Use Authorization (EUA) on November 9th 2020 in COVID-19 patients at risk of progression of the mild-to-moderate disease to a more severe form [9]. Six months later, the FDA included pregnancy as a qualifying condition for the use of MOABs, supported by the National Institutes of Health, though there continues to be very limited data looking at the long-term efficacy and potential risk of poor maternal/fetal outcomes [12].

SARS-CoV-2 variant B.1.1.529 (named Omicron) was first reported to the WHO on November 24th 2021 and was designated a variant of concern (VOC) on November 26th 2021. Since December 26th 2021, the Omicron variant has accounted for more than $90 \%$ of the COVID-19 cases in the USA [10]. Until then, there were three MOABs that were widely available for outpatient management of coronavirus patients. On January 24th 2022, the FDA updated EUA factsheets to discontinue the use of the other two, leaving sotrovimab as the only approved MOAB to be used on COVID-19 positive outpatient management [13-15].

Managing COVID-19 in pregnant patients has imposed a big challenge, especially due to absence of largescale clinical trials. Previously, only a few retrospective case reports and case series have been published with experience in using banlanivimab/etesevimab and casirivimab/imdevimab in pregnant women [16-20]. They have generally shown favorable outcomes without significant side effects. Only Chang and Richley reported adverse reactions during infusion of MOAB, in one and two patients respectively $[19,20]$. 
Sotrovimab is a pan-sarbecovirus monoclonal antibody. Its efficacy against COVID-19 in high risk patients has been studied in COMET-ICE (a Phase III, multi-center, double-blinded, placebo-control trial) and proven to reduce risk of progression of mild to moderate infection to severe form. The study, however excluded any women that were pregnant, breast-feeding or had childbearing potential (lack of effective contraception). To this day (February 20,2022), safe and successful use of sotrovimab has only been reported in one case report in a pregnant patient (with kidney transplant) [11].

\section{Conclusions}

Our patient was pregnant and unvaccinated against COVID-19, which are well-known risk factors for progression of mild to moderate coronavirus to severe form and for need for hospitalization. The outcome of the sotrovimab infusion in our patient was favorable, with rapid resolution of symptoms, prevention of hospitalization and prevention of progression to severe infection. However, there continues to be a dire need for larger-scale studies to derive definitive short and long-term benefits in this patient population.

\section{Additional Information \\ Disclosures}

Human subjects: Consent was obtained or waived by all participants in this study. Conflicts of interest: In compliance with the ICMJE uniform disclosure form, all authors declare the following: Payment/services info: All authors have declared that no financial support was received from any organization for the submitted work. Financial relationships: All authors have declared that they have no financial relationships at present or within the previous three years with any organizations that might have an interest in the submitted work. Other relationships: All authors have declared that there are no other relationships or activities that could appear to have influenced the submitted work.

\section{References}

1. Data on COVID-19 during Pregnancy: Severity of Maternal Illness . (2022). Accessed: February 20, 2022: https://covid.cdc.gov/covid-data-tracker/\#pregnant-population.

2. Lokken EM, Huebner EM, Taylor GG, et al.: Disease severity, pregnancy outcomes, and maternal deaths among pregnant patients with severe acute respiratory syndrome coronavirus 2 infection in Washington State. Am J Obstet Gynecol. 2021, 225:1-14. 10.1016/j.ajog.2020.12.1221

3. Khan DS, Pirzada AN, Ali A, Salam RA, Das JK, Lassi ZS: The differences in clinical presentation, management, and prognosis of laboratory-confirmed COVID-19 between pregnant and non-pregnant women: a systematic review and meta-analysis. Int J Environ Res Public Health. 2021, 18:10.3390/ijerph18115613

4. Allotey J, Stallings E, Bonet M, et al.: Clinical manifestations, risk factors, and maternal and perinatal outcomes of coronavirus disease 2019 in pregnancy: living systematic review and meta-analysis. BMJ. 2020, 370:m3320. 10.1136/bmj.m3320

5. DeSisto CL, Wallace B, Simeone RM, Polen K, Ko JY, Meaney-Delman D, Ellington SR: Risk for stillbirth among women with and without COVID-19 at delivery hospitalization - United States, March 2020September 2021. MMWR Morb Mortal Wkly Rep. 2021, 70:1640-5. 10.15585/mmwr.mm7047e1

6. Dougan M, Nirula A, Azizad M, et al.: Bamlanivimab plus etesevimab in mild or moderate Covid-19. N Engl J Med. 2021, 385:1382-92. 10.1056/NEJMoa2102685

7. Weinreich DM, Sivapalasingam S, Norton T, et al.: REGEN-COV antibody combination and outcomes in outpatients with Covid-19. N Engl J Med. 2021, 385:e81. 10.1056/NEJMoa2108163

8. Gupta A, Gonzalez-Rojas Y, Juarez E, et al.: Early treatment for Covid-19 with SARS-CoV-2 neutralizing antibody sotrovimab. N Engl J Med. 2021, 385:1941-50. 10.1056/NEJMoa2107934

9. Coronavirus (COVID-19) Update: FDA Authorizes Monoclonal Antibody for Treatment of COVID-19 . (2020). Accessed: February 18, 2022: https://www.fda.gov/news-events/press-announcements/coronavirus-covid19-update-fda-authorizes-monoclonal-antibody-tr....

10. COVID Data Tracker: Variant Proportions. (2022). Accessed: February 20, 2022: https://covid.cdc.gov/coviddata-tracker/\#variant-proportions.

11. AlKindi F, Chaaban A, Al Hakim M, Boobes Y: Sotrovimab use for COVID-19 infection in pregnant kidney transplant recipient. Transplantation. 2022, 10.1097/TP.0000000000004083

12. COVID-19 treatment guidelines: anti-SARS-CoV-2 monoclonal antibodies. (2021). Accessed: February 20, 2022: https://www.covid19treatmentguidelines.nih.gov/anti-sars-cov-2-antibody-products/anti-sars-cov-2monoclonal-antibodies/.

13. Bamlanivimab/etesevimab. (2022). Accessed: February 20, 2022: https://www.phe.gov/emergency/events/COVID19/investigation-MCM/Bamlanivimabetesevimab/Pages/default.aspx.

14. REGEN-COV. (2022). Accessed: February 20, 2022: https://www.phe.gov/emergency/events/COVID19/investigation-MCM/cas_imd/Pages/default.aspx.

15. Fact sheet for healthcare providers emergency use authorization (EUA) of sotrovimab . (2022). Accessed: February 20, 2022: https://www.fda.gov/media/149534/download.

16. Hirshberg JS, Cooke E, Oakes MC, Odibo AO, Raghuraman N, Kelly JC: Monoclonal antibody treatment of symptomatic COVID-19 in pregnancy: initial report. Am J Obstet Gynecol. 2021, 225:688-9. 10.1016/j.ajog.2021.08.025

17. Mayer C, VanHise K, Caskey R, Naqvi M, Burwick RM: Monoclonal antibodies casirivimab and imdevimab in pregnancy for coronavirus disease 2019 (COVID-19). Obstet Gynecol. 2021, 138:937-9.

10.1097/AOG.0000000000004603 


\section{Cureus}

18. Thilagar BP, Ghosh AK, Nguyen J, et al.: Anti-spike monoclonal antibody therapy in pregnant women with mild-to-moderate coronavirus disease 2019 (COVID-19). Obstet Gynecol. 2022,

10.1097/AOG.0000000000004700

19. Chang MH, Cowman K, Guo Y, Bao H, Bernstein PS, Gendlina I, Nori P: A real-world assessment of tolerability and treatment outcomes of COVID-19 monoclonal antibodies administered in pregnancy. Am J Obstet Gynecol. 2022, 10.1016/j.ajog.2022.01.018

20. Richley M, Rao RR, Afshar Y, et al.: Neutralizing monoclonal antibodies for coronavirus disease 2019 (COVID-19) in pregnancy: a case series. Obstet Gynecol. 2022, 139:368-72. 10.1097/AOG.0000000000004689 